\title{
Correlation between the methylation of the FUT1 promoter region and FUT1 expression in the duodenum of piglets from newborn to weaning
}

\author{
Chaohui Dai $^{1} \cdot$ Li Sun $^{1} \cdot$ Riwei Xia $^{1} \cdot$ Shouyong Sun ${ }^{1}$. \\ Guoqiang $\mathrm{Zhu}^{2} \cdot$ Shenglong $\mathrm{Wu}^{1} \cdot$ Wenbin Bao ${ }^{1}$
}

Received: 12 July 2016/Accepted: 10 July 2017/Published online: 15 July 2017

(C) Springer-Verlag GmbH Germany 2017

\begin{abstract}
Alpha-(1,2)-fucosyltransferase (FUT1) gene has some influence on economically important traits and disease resistance. DNA methylation plays an important role in human diseases but is relatively poorly studied in pigs by regulating the mRNA expression of genes. The aim of this study was to analyze the influence of promoter methylation on the expression of FUT1 gene. We used bisulfite sequencing PCR (BSP) and qPCR to analyze the methylation of the FUT1 5 -flanking region and FUT1 mRNA expression in the duodenum of Sutai piglets from newborn to weaning. FUT1 contains three $\mathrm{CpG}$ islands upstream of the start codon, of which two are located in the putative promoter region containing multiple promoter elements and transcription factor binding sites, such as $\mathrm{CpG}$ islands, a CAAT box, SP1, and EARLY-SEQ 1. The CpG island between nucleotides -1762 and -580 had a low degree of methylation, and its methylation level was significantly lower in 35-day-old piglets than 8- and 18-day-old piglets $(P<0.05)$. FUT1 mRNA expression was significantly higher in 35-day-old piglets than 8- and 18-day-old piglets $(P<0.05)$. Pearson's correlation analysis showed that the methylation of the $\mathrm{CpG}$ island between nucleotides -1762
\end{abstract}

Electronic supplementary material The online version of this article (doi:10.1007/s13205-017-0880-9) contains supplementary material, which is available to authorized users.

Wenbin Bao

wbbao@yzu.edu.cn

1 Department of College of Animal Science and Technology, Key Laboratory for Animal Genetics, Breeding,

Reproduction and Molecular Design of Jiangsu Province, Yangzhou University, Yangzhou, Jiangsu, People's Republic of China

2 Department of College of Veterinary Medicine, Yangzhou University, Yangzhou, Jiangsu, People's Republic of China and -580 of $F U T 1$ was significantly, negatively correlated with FUT1 mRNA expression $(P<0.05)$. These results demonstrate that differential methylation of $\mathrm{CpG}$ islands negatively regulates the expression of FUT1 in the porcine duodenum, suggesting a probable influence on the resistance of piglets to infection with ETEC F18.

Keywords Pig $\cdot$ FUTl Promoter methylation - BSP

\section{Introduction}

Enterotoxigenic Escherichia coli is the pathogen most frequently responsible for diarrhea and edema in newborn and weaned piglets, and has become a major threat in the pig farming industry (Boldin 2008). Currently, the ETEC strain F18 is one of the most common and most hazardous E. coli pathogens in the pig farming industry (Van den Broeck et al. 2000). Genetic studies have revealed that the adhesion-mediated adhesion and colonization ability of ETEC F18 bacteria in piglets is dependent upon the presence of appropriate receptors for F18 cohesin in the brush borders on epithelial cells in the piglet intestine; when the F18 strain is bound to these receptors, the toxin produced by the bacteria can induce diarrhea and edema in pigs (Bertschinger and Pohlenz 1983; Imberechts et al. 1996). Using a candidate gene approach and linkage analysis, Vogeli et al. (1997) discovered that the $\alpha$-(1,2) fucosyltransferase gene 1 (FUT1) is a candidate gene which can control the adhesion to ETEC F18 receptor. In a study in pigs in Switzerland, Meijerink et al. (1997) reported that the variation of FUT1 gene on M307 locus is correlated with resistance to ETEC F18, and successfully achieved resistance to ETEC F18 by breeding pigs with this marker. FUT1 and its M307 locus are one of the few resistance 
genes and resistance markers for controlling the expression of the receptor for ETEC F18 to be identified in pigs to date. Studies have shown that porcine FUT1 has some influence on economically important traits and disease resistance (Bao et al. 2012a, b; Zhang et al. 2007). Based on this research, we and other Chinese researchers have systematically analyzed the FUT1-coding region in nearly 30 Chinese native pig breeds and foreign pig breeds. The variation of FUT1 gene on M307 locus, which has been used as a genetic marker in foreign breeds, displays an extremely skewed distribution in native Chinese pig breeds compared to foreign breeds (Bao et al. 2008; Yan et al. 2003), and the presence or absence of the M307 locus does not significantly influence the expression level of FUT1 (Bao et al. 2012a, b). This makes the development of resistance to ETEC F18 in local Chinese pig breeds difficult and problematic. Therefore, screening for other candidate ETEC F18 resistance genes in local Chinese pig breeds is required, and additionally, the mechanism by which the non-coding regions of the FUT1 gene, especially the promoter region, offer resistance to ETEC F18 urgently requires investigation.

DNA methylation, a critical component of epigenetic control, plays an important role in cell differentiation, embryonic development, gene regulation, genomic imprinting, and X-chromosome inactivation (Reik et al. 2001; Ehrlich 2003; Li 2002; Bird 2002; Li et al. 1993; Heard 2004). Sullivan et al. (2012) revealed that hypermethylation of the insulin-like growth factor binding protein 7 (IGFBP7) promoter can lead to gene silencing. Many human diseases, such as coronary artery disease, Alzheimer's disease, and cancer, are closely linked to altered promoter methylation (Kim et al. 2011; Friso et al. 2012; Silva et al. 2008); however, reports of the association between diseases and altered promoter methylation in pigs are relatively rare. Kang et al. (2007) suggested that dynamic methylation changes may play an important role in the establishment and maintenance of tissue-specific gene expression in cloned pigs. Xiao et al. (2012) analyzed the promoter methylation status and gene expression levels of the tyrosine protein kinase lyn $(L Y N)$ gene and demonstrated that methylation plays an important role in the regulation of porcine $L Y N$ gene transcription. Ling et al. (2009) revealed that the methylation status of the porcine adiponectin gene promoter dynamically fluctuates in a manner which correlates with adiponectin gene expression during development.

The promoter, an integral upstream regulatory region of a gene, controls the initiation of gene transcription as well as the gene expression level. The promoter regulates gene expression through a number of mechanisms, including promoter methylation (Juven-Gershon and Kadonaga 2010). Thus, intensive study of promoter methylation is essential to fully understand the mechanisms of gene regulation. As FUT1 is the most important candidate gene for the breeding of porcine ETEC F18 disease resistance, research on the potential methylation modifications which the FUT1 promoter undergoes will provide a new approach for investigating the mechanisms by which FUT1 confers resistance to ETEC F18. Nevertheless, data on the porcine FUT1 5'-flanking are absent in the authoritative database (GenBank); therefore, it is difficult to initiate studies on the FUT1 promoter. In a previous study, we cloned the FUTI promoter sequence (using duodenum DNA of Sutai piglets by Genome Walking) and compared the sequences (see sequence $U 70883.2$ in GenBank) via a high-throughput approach and by Blastn analysis. We retrieved the sequence 2000 bp upstream of the start codon of FUT1. In this study, we investigated the methylation status of the FUT1 5'-flanking and explored the influence of FUT1 promoter methylation on the gene expression levels of FUT1 in the duodenum of Sutai piglets from the newborn stage to weaning (8, 18, and 35 days old). This study provides a foundation for further studies of the mechanisms regulating porcine $F U T 1$ expression.

\section{Materials and methods}

\section{Experimental reagents}

The EpiTect Bisulfite Kit was purchased from Qiagen (Valencia, CA, USA), TRIzol reagent from Invitrogen (Carlsbad, CA, USA), and Taq DNA polymerase, dNTPs, PCR purification kit, Plasmid DNA Extract kit, Competent Escherichia coli cell, pMD18-T vector, and SYBR Green Real-time PCR Mix from TaKaRa (Dalian, China). All the other chemicals were of analytical reagent grade.

\section{Bioinformatic analysis and primer design}

Analysis and identification of the $\mathrm{CpG}$ islands, transcription initiation site, and putative promoter region in the $5^{\prime}$ flanking of FUT1 were performed using the online tools MethPrimer (http://www.urogene.org/methprimer/index1. html), NNPP (http://www.fruitfly.org/seq_tools/promoter. html), PROMOTER 2.0 (http://www.cbs.dtu.dk/services/ Promoter), FirstEF (http://rulai.cshl.org/tools/FirstEF/), and TFSEARCH (http://www.cbrc.jp/research/db/ TFSEARCH.html). Based on this analysis and the location of the predicted $\mathrm{CpG}$ islands, bisulfite-sequencing PCR (BSP) primers were designed using Methyl Primer 
Table 1 Primers for analysis of FUT1 methylation

\begin{tabular}{llll}
\hline Primer & Sequence & Length (bp) & Location \\
\hline FUT1-1 & F:5'-GATTTAGGTGTTTTTGGTTTGGAAG-3' & 441 & -2174 to -1823 \\
& R:5'-CCCTCTCCAAAAATCTAACCCTC-3' & & \\
FUT1-2 & F:5'-GGTTAGATTTTTGGAGAGGGAG-3' & 291 & -1842 to -1552 \\
& R:5'-AAACRCTAAAATTACACTTAACTCTC-3' \\
FUT1-3 & F:5'-TTTTTGGAGAGTTAAGTGTAATTTTAG-3' & 280 & -1583 to -1304 \\
& R:5'-CTCCTTCCRACRAAATCCACT-3' & & -1343 to -906 \\
FUT1-4 & F:5'-GAGAYGTGYGTGTTTAGAAAGTGGAT-3' & & \\
FUT1-5 & R:5'-CTAACTACCCAACAACCACATCCCTC-3' & & -1010 to -580 \\
& F:5'-AAGGGAAGAGGGAGAGGTTG-3' & 431 & \\
\hline
\end{tabular}

Both $\mathrm{R}$ and $\mathrm{Y}$ are degenerate bases

$R$ stands for $\mathrm{A} / \mathrm{G}, Y$ stands for $\mathrm{C} / \mathrm{T}$

Express 1 software (Table 1). All primers were synthesized by Sangon (Shanghai, China).

\section{Experimental animal}

Sutai pig is a new breed of high-quality lean-meat-type pig bred by Sutai pig breeding center in Suzhou city. It is a breed developed from a Duroc $(50 \%) \times$ Meishan $(50 \%)$ cross after 15 years of practice. In 1999, it was approved by National Committee of Livestock and Poultry Species for a new variety. All the pigs (Sutai pigs, 8, 18, and 35 days old, $n=5$ each) included in the experiment were healthy and were, respectively, selected from five different families, with same feeding condition, similar birth weights, weaning weights, and body sizes. The pigs were purchased from Suzhou Sutai Pig Breeding Center (Suzhou, China) and all experiments were conducted at the Animal Hospital of Yangzhou University according to the regulation for the Administration of Affairs Concerning Experimental Animals (Ministry of Science and Technology, China, revised in June 2012) and approved by the experimental animal using permit with Permit No. SYXK (Su) 2012-0029.

\section{Real-time PCR analysis}

RNA was isolated from the duodenal tissues of the Sutai pigs using TRIzol reagent, according to the manufacturer's instructions. Single-stranded cDNA was generated using the PrimeScript RT-PCR Kit following the manufacturer's directions. Real-time quantitative PCR was performed using an ABI Prism 7500 sequence-detection system (Applied Biosystems, Foster City, CA, USA) with SYBR Green PCR Master Mix, according to the manufacturer's instructions. The primer sequences for FUTl were: F,
5'-TTTTAAGCCCCCAAACTGCC-3' and R, 5'-TAAAT CGACCCCATCAGCCTC-3'. The GAPDH primers used as internal control and thermocycler conditions are described in our previous study (Bao et al. 2012a, b), the expression of FUT1 in each sample was normalized to $G A P D H$. Triplicate PCR amplifications were performed for each sample. The fold change in relative gene expression was calculated using the standard $2^{-\Delta \Delta \mathrm{Ct}}$ method (Livak and Schmittgen 2001).

\section{Promoter methylation analysis}

Genomic DNA was extracted from porcine duodenal tissues by standard phenol/chloroform extraction and subjected to bisulfite conversion using the EpiTect Bisulfite Kit, according to the manufacturer's instructions. Touchdown PCR was used to amplify the bisulfite-treated DNA (BST-DNA). The $50-\mu \mathrm{L}$ reactions included $3.0 \mu \mathrm{L}$ of DNA template, $3 \mu \mathrm{L}$ of $10 \times$ PCR buffer, $2 \mu \mathrm{L}$ of $\mathrm{Mg}^{2+}$ (25 mmol), $1 \mu \mathrm{L}$ of forward primer $(10 \mu \mathrm{M}), 1 \mu \mathrm{L}$ of reverse primer $(10 \mu \mathrm{M}), 1 \mu \mathrm{L}$ of dNTPs $(10 \mathrm{mmol}), 0.8 \mu \mathrm{L}$ of $\mathrm{Taq}$ polymerase $(5 \mathrm{U} / \mu \mathrm{L})$ and $38.2 \mu \mathrm{L}$ of water. The following reaction conditions were used: $98{ }^{\circ} \mathrm{C}$ for $4 \mathrm{~min}$, then 20 cycles of $94{ }^{\circ} \mathrm{C}$ for $45 \mathrm{~s}, 66{ }^{\circ} \mathrm{C}$ for $45 \mathrm{~s}$ (which reduced by $0.5^{\circ} \mathrm{C}$ each cycle), and $72{ }^{\circ} \mathrm{C}$ for $1 \mathrm{~min} ; 20$ cycles of $94{ }^{\circ} \mathrm{C}$ for $45 \mathrm{~s}, 56{ }^{\circ} \mathrm{C}$ for $45 \mathrm{~s}$ and $72{ }^{\circ} \mathrm{C}$ for $1 \mathrm{~min}$, and a final extension at $72{ }^{\circ} \mathrm{C}$ for $10 \mathrm{~min}$. The PCR products were subjected to electrophoresis on agarose gels, excised, purified, and inserted into the pMD18-T vector. The recombinant clones were used to transform E.coli TB1 cells. The positive recombinant clones were selected on LB agar plates containing $100 \mu \mathrm{g} / \mathrm{mL}$ ampicillin and confirmed by PCR and DNA sequencing (five positive recombinant clones were selected from each individual). 
The methylation rate was calculated as the number of $\mathrm{CpG}$ methylated loci/number of $\mathrm{CpG}$ loci.

\section{Results}

\section{Analysis of the CpG islands, promoter elements, and transcription factor binding sites in the $5^{\prime}$ - flanking of porcine $F U T 1$}

Analysis of the FUT1 gene $5^{\prime}$-flanking region (2000 bp upstream of the initiation codon) revealed that porcine FUT1 has an intron-cut $5^{\prime}$-flanking and FUTl contains only one intron (Fig. 1). CpG islands in the porcine FUT1 $5^{\prime}$ flanking were identified using the online software MethPromoter and Methyl Promoter Express software. The FUT1 5'-flanking contains three $\mathrm{CpG}$ islands: $\mathrm{CpG}$ island 1 is located between nucleotides -1779 and -886 with a length of $894 \mathrm{bp}, \mathrm{CpG}$ island 2 is located between nucleotides -796 and -619 with a length of $178 \mathrm{bp}$, and $\mathrm{CpG}$ island 3 is located between nucleotides -316 and -130 with a length of $187 \mathrm{bp}$. CpG islands 1 and 2 are located within the putative promoter region and $\mathrm{CpG}$ island 3 is located within intron 1 (Fig. 1). Online analysis using the tools NNPP, PROMOTER 2.0, FirstEF, and TFSEARCH showed that the putative FUT1 promoter region contains multiple promoter element characteristics and transcription factor binding sites, including $\mathrm{CpG}$ islands, a CAAT box, EARLY-SEQ 1, T-Ag, PuF, and SP1 (Fig. 2).
-1604 GGTGCAGCCGTARAARASARTCTCTGGAGAGTCAAGTGCAATCCAGCG

-1554 TCTTTGCTTTGTAGAATGACAATGGTCCGACCGCCOGGGCTAGGGTGGT

Chit box

PuF

-1504 GCGGAGCGGCCTTGGGCAAGGAAACTGCTTCGGGTCTGCCGAAGGGCACG

-1454 GCCTTTGTTCCAGASGCCGGCTGCAGCCCGGGCCTCTGCGGGAGGGGCAG

$\mathrm{T}-\mathrm{Ag}$

1404 GAGAGATCCACTTCCACCTCGGCTCTCGCCGTTCCCGCCCTGGGAGAGG

EARLY-SEQ1

-1354 CTGOGGCCTCCGAGACGTGOGTGTCTAGAMAGTGGATTCOCCOGGAMGA
-1304 GAAGCOGGGACCCGGACATTTTGCGGGCGGAGGGGTCGATCGGCGGCC

SP1

-1254 CCGGTGCGCC AGGCGCGCTCACGCCGGACCCGGAGAGCTGGGGAAGGCCT

-1204 CGCATCCGCTTGCGGAGGAGCCTGGCGAACAGGTTCTGC TGCCCCCGGGG

-1154 AGGACTCGGCAGGGGGCGGGGGGCCGCGCACGACGCCTGGACCGGGTGT

BTEB4

NF-kappaB1

\section{-1104 CCCCCGCGTCCCCGGCGTCCCTACCCAAGCCGCTGGGGCGTGGCGGCACT}

-1054 CGGCGCATCCAGGTACARGGGCGGGTGGCGAACCAAGAASCTGGAAGGGA

Fig. 2 Prediction of cis-acting elements and transcription factor binding sites in the $5^{\prime}$-flanking region of porcine FUT1. In this core promoter region, the $\mathrm{CpG}$ island sequence was in the box and the non$\mathrm{CpG}$ island sequence was in other area. All CG sites were highlighted with a yellow background and all methylated $\mathrm{C}$ sites were highlighted in bold red font. The underline represents a possible transcription factor binding site

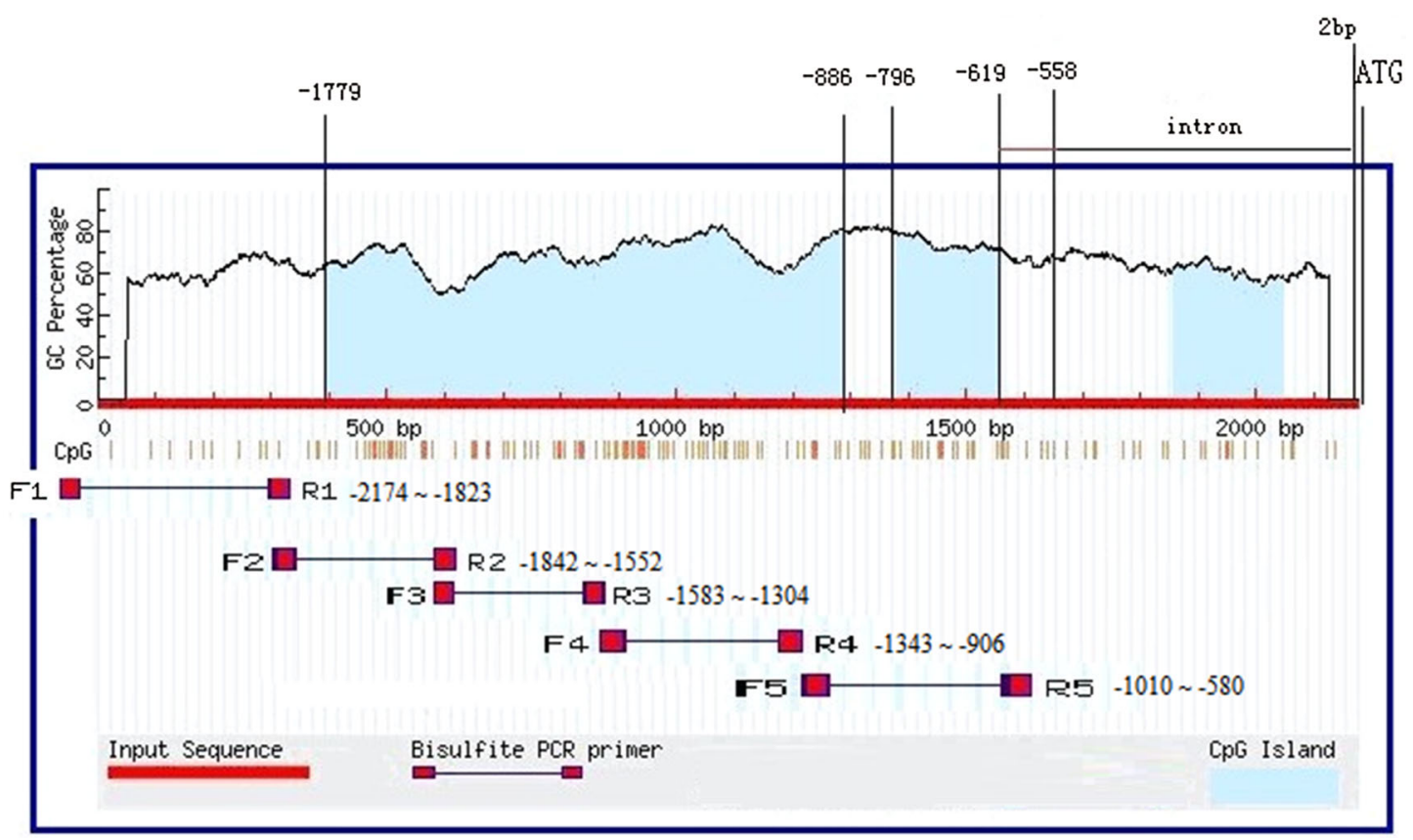

Fig. 1 Structure of the $5^{\prime}$-flanking region of porcine FUT1 showing the position of the $\mathrm{CpG}$ islands and primers for bisulphite sequencing PCR 
Analysis of the methylation status of the putative promoter region of porcine $F U T 1$

Five primer pairs were designed to examine the levels of methylation in the FUT1 5'-flanking (Fig. 1). The products produced by each primer pair from the amplification of cDNA extracted from the pig duodenum were examined by $1 \%$ agarose gel electrophoresis. The size of the amplified fragments corresponded with the expected product sizes for each primer pair and each primer pair amplified a single specific product which could be directly cloned and sequenced (Supplementary Fig. 1).

The methylation status of the putative FUT1 promoter region was examined in the duodenum of piglets of three different ages. As shown in Fig. 3 and Table 2, CpG methylation sites were ubiquitously present in the porcine putative FUT1 promoter region. The non-CpG island (from -2174 to -1762 ) was highly methylated, with an average methylation rate of $74.81 \%$. The methylation status of the non-CpG island in the putative $F U T 1$ promoter region was not significantly different among the three ages of piglets tested. The $\mathrm{CpG}$ island in the putative FUT1 promoter region (from -1762 to -580 ) had a low degree of methylation, with an average methylation rate of $3.82 \%$. Although the degree of methylation of the $\mathrm{CpG}$ island in the putative FUT1 promoter region was low, its methylation status varied with the age of the piglets: the degree of methylation in the duodenum of 8-day-old piglets was $4.1 \%$, which increased slightly $(4.79 \%)$ but non-significantly compared to 18-day-old piglets. The degree of methylation drastically reduced in 35-day-old piglets $(2.56 \%)$ and was significantly lower than that in 18-day-old and 8-day-old piglets $(P<0.05)$.

\section{Correlation between the methylation status of the putative promoter region and the expression levels of porcine $F U T 1$}

Quantitative real-time PCR was used to measure the expression of FUT1 in the duodenum of piglets at three
Fig. 3 Analysis of the methylation status of the FUT1 $5^{\prime}$-flanking region in the duodenum of piglets of different ages. Circles CpGs, black circles methylated CpGs, and white circles unmethylated CpGs; FUT1-1 to 5 the five primer pairs for bisulfite sequencing PCR. Every circle represents a $\mathrm{CG}$, black circles methylated $\mathrm{CG}$, and white circles unmethylated CG

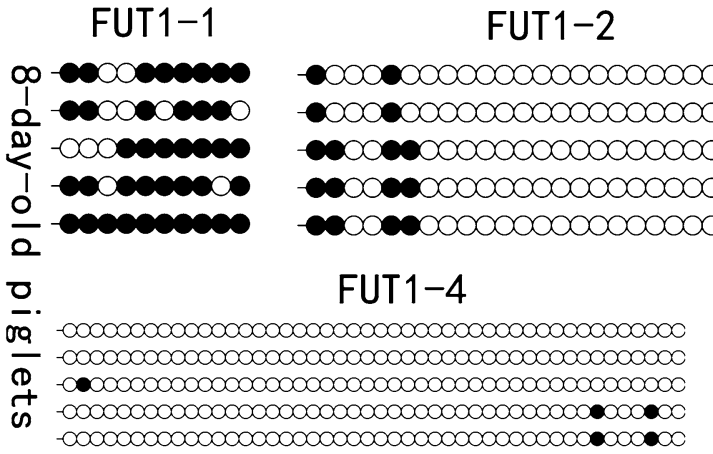

FUT1-1

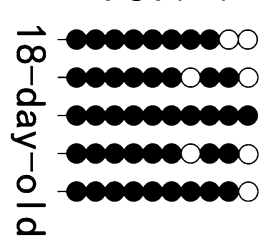

ㅁ. 1000000000000000000000000000000000000000000000

val -0000000000000000000000000000000000000000000000

- -000000000000000000000000000000000000000000000

I) -0000000000000000001000000000000000000000000000

if -0000000000000000000100000000000000000000000000

\section{FUT1-1}

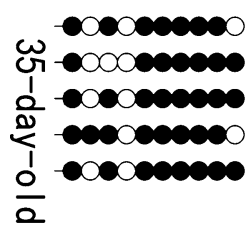

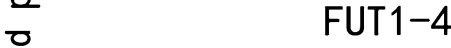

o.- -0000000000000000000000000000000000000000000000

10 - 0000000000000000000000000000000000000000000000

I. -0000000000000000000000000000000000001000000000

is -0000000000000000000000000000000000000000000000

$-0000000000000000000000000000000000000000000$

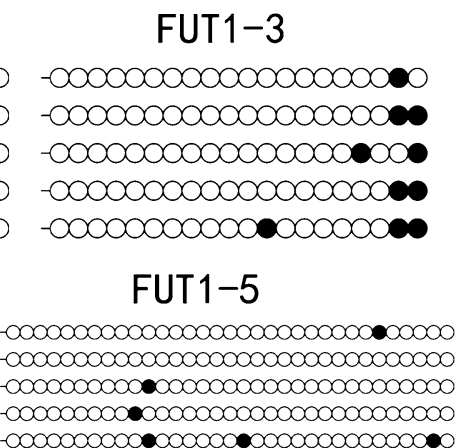

$-000000000000000000000000000000000$ $-000000000000000000000000000000000$ $-000000000100000000000000000001000$ $-000000000100000000000000000001000$ $-000000000100000000000000000001000$

FUT1-3

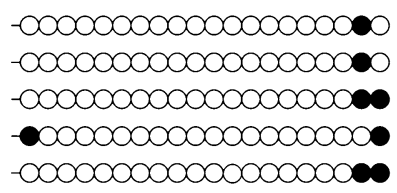

FUT1-5

$-000000000000000000000000000000000$ $-000000000000000000000000000000000$ $-000000000000000000000000000000000$ -00000000001000000 000000000000000 $-00000000000000000000000000000000$

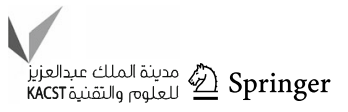


Table 2 Methylation status and expression level of the FUT1 gene in piglets of different ages

\begin{tabular}{lcccc}
\hline & Age (number) & & & \\
\cline { 2 - 5 } & 8 days $(n=5)$ & 18 days $(n=5)$ & 35 days $(n=5)$ & Mean \\
\hline Methylation of non-CpG islands $(\%)$ & $78.53 \pm 15.26$ & $76.67 \pm 9.55$ & $81.11 \pm 2.72$ & $74.81 \pm 8.87$ \\
Methylation of CpG islands (\%) & $4.50 \pm 1.76^{\mathrm{a}}$ & $4.79 \pm 1.99^{\mathrm{a}}$ & $2.56 \pm 1.52^{\mathrm{b}}$ & $3.82 \pm 1.88$ \\
FUT1 mRNA expression & $20.88 \pm 8.69^{\mathrm{a}}$ & $16.057 \pm 6.241^{\mathrm{a}}$ & $66.12 \pm 14.93^{\mathrm{b}}$ & $37.07 \pm 13.377$ \\
\hline
\end{tabular}

Values with different superscripts within the same line differ significantly $(P<0.05)$

different ages. The relative expression level of FUT1 decreased between 8-day-old and 18-day-old and then increased to the highest levels in 35-day-old piglets $(P<0.05$; Table 2). Pearson's correlation analysis indicated that the methylation status of the $\mathrm{CpG}$ island in the putative promoter region significantly, negatively correlated with the expression level of FUTl in the duodenum as the piglets aged (Pearson's correlation coefficient is $-0.999, P<0.05)$. There was no significant correlation between the methylation status of the non- $\mathrm{CpG}$ island in the putative promoter region and the expression of FUTI in the porcine duodenum (Pearson's correlation coefficient is $0.545, P>0.05)$.

\section{Discussion}

FUT1 controls the expression of ETEC F18 and is one of the few candidate anti-diarrhea genes to have been identified in piglets (Vogeli et al. 1997; Meijerink et al. 1997). Many studies have studied the coding region of porcine FUT1; however, few studies have focused on the FUT1 5'flanking region or promoter region due to an absence of sequence data on this region in authoritative databases. In a previous study, we retrieved the sequence of the region 2000 bp upstream of the FUT1 start codon using gene walking and high-throughput comparison (see sequence U70883.2 in GenBank), which provided a basis for further experiments. Promoter regions can be divided into $\mathrm{CpG}$ islands and non- $\mathrm{CpG}$ islands according to their $\mathrm{CpG}$ content. In this study, we first analyzed the 5 '-flanking of FUT1 and found that the putative promoter region contains one non-CpG island ( -2174 to -1762$)$ and one $\mathrm{CpG}$ island $(-1762$ to -580$)$.

Suzuki and Bird (2008) reported that the highly dynamic process of promoter methylation is an important regulation mechanism for normal developmental processes. Such dynamic methylation processes are particularly evident during embryonic development (Santos et al. 2002) and methylation plays a key role in the reprogramming of the genome in the early mammalian embryo (Thurston et al. 2007; Haaf 2006). In the present study, we observed that the methylation status of the putative promoter region of FUT1 altered dynamically as the piglets aged, with a large difference between the non- $\mathrm{CpG}$ island and $\mathrm{CpG}$ island. From the newborn stage to the weaning period in piglets, the non-CpG island remained highly methylated and its methylation status did not change significantly. The methylation status of the non-CpG island did not significantly correlate with the FUTI expression level in the duodenum. This indicates that although the non- $\mathrm{CpG}$ island has a high degree of methylation, its methylation status does not significantly affect FUT1 expression. The CpG islands in the putative FUT1 promoter region had a low degree of methylation and its degree of methylation varied remarkably as the piglets aged; the degree of methylation in 35-day-old piglets was significantly lower than 8- and 18-day-old piglets. Real-time PCR demonstrated that the FUT1 expression level was significantly higher in 35-dayold piglets than 8- and 18-day-old piglets and the degree of methylation in the $\mathrm{CpG}$ islands was significantly, negatively correlated with the FUT1 mRNA expression level. This indicates that methylation of the $\mathrm{CpG}$ island in the putative $F U T 1$ promoter region negatively regulates $F U T 1$ gene expression.

Pigs of different ages usually display different degrees of sensitivity to disease, for example, newborn piglets are vulnerable to infection with $E$. coli strain $\mathrm{K} 88$, whereas 35-day-old piglets are susceptible to infection with ETEC F18 (Jones and Rutter 1972; Verdonck et al. 2002). In the present study, we observed that expression level of FUTI was significantly higher in the duodenum of 35-day-old piglets than 8-day-old piglets, suggesting that the FUT1 expression level is associated with the susceptibility to infection by ETEC F18. Our preliminary studies and other studies suggest that the FUT1 expression level directly influences the sensitivity of piglets to ETEC F18 (Meijerink et al. 1997; Bao et al. 2012a, b); these findings suggest that methylation of the $\mathrm{CpG}$ island in the putative FUT1 promoter region may affect the resistance of piglets 
to ETEC F18. Besides, we found that methylation of $\mathrm{CpG}$ in FUT1-2 was a little different in 8 days, 18 days, and 35 days, which suggests that specific $\mathrm{CpG}$ sites may contribute more than others to regulate FUT1 mRNA expression. However, we found that these methylated CG sites were not located in the transcription factor binding sites of the core promoter region of FUT1 gene, so how they regulated the expression of FUT1 gene needed further studies.

In a previous study, we preliminarily explored the mechanisms regulating FUT1 expression and analyzed the expression level of the FUT1 M307 locus in different genotypes of Sutai pigs. The results showed that the mutation of the M307 locus (G/A) does not have a significant influence on the expression of FUT1 (Bao et al. $2012 a, b)$. The results of this study further suggest that the regulatory mechanism controlling the response to ETEC F18 may possibly involve altered methylation of the $\mathrm{CpG}$ islands in the putative $F U T 1$ promoter region.

There are two mechanisms by which promoter methylation affects gene regulation. First, the methylation of essential cis-acting elements in the promoter region influences the binding of transcription factors (Kass et al. 1997). Second, co-suppression factor complexes, including histone deacetylase, are recruited by the methylated cis-acting elements via methyl-CpG-binding proteins, which inhibit transcription (Nan et al. 1998; Magnaghi-Jaulin et al. 1998). Our bioinformatic analysis indicates that the putative FUT1 promoter region contains a number of transcription factor binding sites, such as EARLY-SEQ 1, PuF, and SP1-binding sites, within the CpG islands. SP1 is a key transcription factor required for mRNA transcription, and SP1-binding sites contain a CpG site (Holler et al. 1988). Methylation of $\mathrm{CpG}$ sites can directly influence the ability of SP1 to bind DNA and influence gene expression; likewise, EARLY-SEQ 1 also contains a CpG site. Methylation of the $\mathrm{CpG}$ sites within these transcription factor binding sites is likely to directly influence the expression of FUT1. Gonzalgo et al. (1998) reported that a certain ratio of $\mathrm{CpG}$ island methylation $(>60 \%)$ can be sufficient to inhibit gene expression, whereas a lower ratio of methylation reduces the transcription and expression of genes. Thus, it is not necessary for all $\mathrm{CpG}$ sites to be methylated to inhibit gene expression; rather a regulatory effect can be achieved by controlling the degree of methylation in the binding sites of several transcription factors.

This study has a number of limitations. The methylation status of FUT1 should be examined in porcine tissues other than the duodenum, and larger numbers of samples should be tested to confirm the relationship between $\mathrm{CpG}$ island methylation and FUT1 expression. Further intensive analysis of the upstream control elements in the putative FUT1 promoter region is required to identify the key methylation sites which regulate FUT1 expression.

\section{Conclusions}

In the piglet duodenum, the low levels of methylation of the $\mathrm{CpG}$ island in the putative FUT1 promoter region significantly, negatively correlate with FUT1 expression as the piglets age. Methylation of the $\mathrm{CpG}$ island in the putative FUT1 promoter region may possibly play a role in regulating the resistance of pigs to ETEC F18.

Acknowledgements This research was funded by the Natural Science Foundation of the Jiangsu Higher Education Institutions of China (14KJA230003), the National Natural Science Funds (31172183), the Genetically Modified Organisms Technology Major Project (No. 2014ZX0800601B), and the Priority Academic Program Development of Jiangsu Higher Education Institutions (PAPD).

\section{Compliance with ethical standards}

Conflict of interest The authors declare that they have no conflict of interest in the publication.

\section{References}

Bao WB, Wu SL, Musa HH, Zhu GQ, Chen GH (2008) Genetic variation at the alpha-1-fucosyltransferase (FUT1) gene in Asian wild boar and Chinese and Western commercial pig breeds. J Anim Breed Genet 125:427-430

Bao WB, Ye L, Pan ZY, Zhu J, Du ZD, Zhu GQ, Huang XG, Wu SL (2012a) The effect of mutation at M307 in FUT1 gene on susceptibility of Escherichia coli F18 and gene expression in Sutai piglets. Mol Biol Rep 39:3131-3136

Bao WB, Ye L, Zhu J, Pan ZY, Zhu GQ, Huang XG, Wu SL (2012b) Evaluation of M307 of FUT1 gene as a genetic marker for disease resistance breeding of Sutai pigs. Mol Biol Rep 39:4223-4228

Bertschinger HU, Pohlenz J (1983) Bacterial colonization and morphology of the intestine in porcine Escherichia coli enterotoxemia (edema disease). Vet Pathol 20:99-110

Bird A (2002) DNA methylation patterns and epigenetic memory. Gene Dev 16:6-21

Boldin B (2008) Persistence and spread of gastro-intestinal infections: the case of enterotoxigenic Escherichia coli in piglets. B Math Biol 70:2077-2101

Ehrlich M (2003) Expression of various genes is controlled by DNA methylation during mammalian development. J Cell Biochem 88:899-910

Friso S, Lotto V, Choi SW, Girelli D, Pinotti M, Guarini P, Udali S, Pattini P, Pizzolo F, Martinelli N, Corrocher R, Bernardi F, Olivieri O (2012) Promoter methylation in coagulation F7 gene influences plasma FVII concentrations and relates to coronary artery disease. J Med Genet 49:192-199

Gonzalgo ML, Hayashida T, Bender CM, Pao MM, Tsai YC, Gonzales FA, Nguyen HD, Nguyen TT, Jones PA (1998) The role of DNA methylation in expression of the p19/p16 locus in human bladder cancer cell lines. Cancer Res 58:1245-1252

Haaf $T$ (2006) Methylation dynamics in the early mammalian embryo: implications of genome reprogramming defects for development. Curr Top Microbiol 310:13-22

Heard E (2004) Recent advances in X-chromosome inactivation. Curr Opin Cell Biol 16:247-255

Holler M, Westin G, Jiricny J, Schaffner W (1988) Sp1 transcription factor binds DNA and activates transcription even when the binding site is CpG methylated. Gene Dev 2:1127-1135

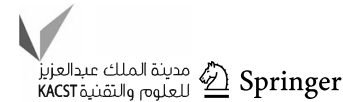


Imberechts H, Wild P, Charlier G, De Greve H, Lintermans P, Pohl P (1996) Characterization of F18 fimbrial genes fedE and fedF involved in adhesion and length of enterotoxemic Escherichia coli strain 107/86. Microb Pathogenesis 21:183-192

Jones GW, Rutter JM (1972) Role of the K88 antigen in the pathogenesis of neonatal diarrhea caused by Escherichia coli in piglets. Infect Immun 6:918-927

Juven-Gershon T, Kadonaga JT (2010) Regulation of gene expression via the core promoter and the basal transcriptional machinery. Dev Biol 339:225-229

Kang JK, Park KW, Chung YG, You JS, Kim YK, Lee SH, Hong SP, Choi KM, Heo KN, Seol JG, Lee JH, Jin DI, Park CS, Seo JS, Lee HW, Han JW (2007) Coordinated change of a ratio of methylated H3lysine 4 or acetylated $\mathrm{H} 3$ to acetylated $\mathrm{H} 4$ and DNA methylation is associated with tissue-specific gene expression in cloned pig. Exp Mol Med 39:84-96

Kass SU, Pruss D, Wolffe AP (1997) How does DNA methylation repress transcription? Trends Genet 13:444-449

Kim DS, Lee JY, Lee SM, Choi JE, Cho S, Park JY (2011) Promoter methylation of the $R G C 32$ gene in nonsmall cell lung cancer. Cancer 117:590-596

Li E (2002) Chromatin modification and epigenetic reprogramming in mammalian development. Nat Rev Genet 3:662-673

Li E, Beard C, Janisch AR (1993) Role for DNA methylation in genomic imprinting. Nature 366:362-365

Ling F, Li JQ, Wang C, Liu C, DU H, Xiao ZZ, Wang LL, Chen YS (2009) Methylation status of pig adiponectin promoter and its expression. Hereditas 31:1013-1019

Livak KJ, Schmittgen TD (2001) Analysis of relative gene expression data using realtime quantitative PCR and the $2^{-\Delta \Delta \mathrm{Ct}}$ method. Methods 25:402-408

Magnaghi-Jaulin AL, Groisman R, Naguibneva I, Robin P, Lorain S, Le Villain JP, Troalen F, Trouche D, Harel-Bellan A (1998) Retinoblastoma protein represses transcription by recruiting a histone deacetylase. Nature 391:601-605

Meijerink E, Fries R, Vögeli P, Masabanda J, Wigger G, Stricker C, Neuenschwander S, Bertschinger HU, Stranzinger G (1997) Two $\alpha(1,2)$ fucosyltransferase genes on porcine chromosome 6q11 are closely linked to the blood group inhibitor (S) and Escherichia coli F18 receptor (ECF18R) loci. Mamm Genome 8:736-741

Nan XS, Ng HH, Johnson CA, Laherty CD, Turner BM, Eisenman RN, Bird A (1998) Transcriptional repression by the methyl-
CpG-binding protein MeCP2 involves a histone deacetylase complex. Nature 393:386-389

Reik W, Dean W, Walter J (2001) Epigenetic reprogramming in mammalian development. Science 293:1089-1093

Santos F, Hendrich B, Reik W, Dean W (2002) Dynamic reprogramming of DNA methylation in the early mouse embryo. Dev Biol 241:172-182

Silva PN, Gigek CO, Leal MF, Bertolucci PH, de Labio RW, Payão SL, Smith Mde A (2008) Promoter methylation analysis of SIRT3, SMARCA5, HTERT and CDH1 genes in aging and Alzheimer's disease. J Alzheimers Dis 13:173-176

Sullivan L, Murphy TM, Barrett C, Loftus B, Thornhill J, Lawler M, Hollywooda D, Lynchd T, Perrya Antoinette S (2012) IGFBP7 promoter methylation and gene expression analysis in prostate cancer. J Urology 188:1354-1360

Suzuki MM, Bird A (2008) DNA methylation landscapes: provocative insights from epigenomics. Nat Rev Genet 9:465-476

Thurston A, Lucas ES, Allegrucci C, Steele W, Young LE (2007) Regionspecific DNA methylation in the preimplantation embryo as a target for genomic plasticity. Theriogenology 68(Suppl 1):S98-S106

Van den Broeck W, Cox E, Oudega B, Goddeeris BM (2000) The F4 fimbrial antigen of Escherichia coli and its receptors. Vet Microbiol 71:223-244

Verdonck F, Cox E, van Gog K, Van der Stede Y, Duchateau L, Deprez P, Goddeeris BM (2002) Different kinetic of antibody responses following infection of newly weaned pigs with an F4 enterotoxigenic Escherichia coli strain or an F18 verotoxigenic Escherichia coli strain. Vaccine 20:2995-3004

Vogeli P, Meijerink E, Fries R, Neuenschwander S, Vorländer N, Stranzinger G, Bertschinger HU (1997) A molecular test for the detection of E. coli F18 receptors: a breakthrough in the struggle against oedema disease and postweaning diarrhoea in swine. Schweiz Arch Tierh 139:479-484

Xiao Z, Wang C, Mo D, Li J, Chen Y, Zhang Z, Cong P (2012) Promoter CpG methylation status in porcine Lyn is associated with its expression levels. Gene 511:73-78

Yan XM, Ren J, Guo YM, Ding NS, Chen KF, Gao J, Ai HS, Chen CY, Ma JW, Huang LS (2003) Research on the genetic variations of al-fucosytransferase (FUT1) gene in 26 pig breeds. Acta Genetica Sinica 30:830-834

Zhang YH, Zhou ZX, Cao GQ (2007) FUT1 gene polymorphism and its correlation with litter size traits. Hereditas 29:52-56 